\title{
La incorporación de la Política Educativa Supranacional sobre la educación inclusiva de las personas con discapacidad en las agendas políticas nacionales de España y los Estados Unidos
}

\author{
Kline, Jorjann Marie \\ Grupo de Investigación sobre Políticas Educativas Supranacionales (GIPES) \\ Universidad Autónoma de Madrid, Madrid, España \\ Jorjann3@gmail.com
}

\section{Resumen}

La comunicación global abierta proporciona acceso a una red compartida de evidencia y experiencia con respecto a las políticas y prácticas en un amplio espectro de asuntos. La cooperación entre individuos, agencias, países, y organizaciones internacionales crea una colección dinámica de información que sirve como banco de conocimiento global. Las políticas educativas supranacionales aportan pautas consolidadas para que los países se adapten a la política nacional. En el campo de la educación, la inclusión de niños con necesidades educativas especiales se ha convertido en una importante discusión global y se establece como un componente esencial de la política educativa. Esta obra pretende analizar la alineación de la política educativa supranacional sobre la educación inclusiva de estudiantes con discapacidades, con las políticas nacionales de educación de España y Estados Unidos, para evaluar la variabilidad entre las políticas y posibilitar recomendaciones para futuras acciones para mejorar de la educación inclusiva. Se incorporan políticas educativas supranacionales de organizaciones internacionales como Organización de las Naciones Unidas para la Educación, la Ciencia y la Cultura (UNESCO), Organización para la Cooperación y el Desarrollo Económicos (OCDE), y la Organización de Naciones Unidas (ONU). Las políticas educativas nacionales se centran en Individuals with Disabilities Education Act (IDEA) en los EEUU y la Ley Orgánica 2/2006, de 3 de mayo, de Educación (LOE) en España. Este estudio compara las políticas educativas nacionales de España y Estados Unidos en los siguientes aspectos: leyes, procesos de educación inclusiva, entornos de inclusión, provisión de servicios interdisciplinarios, diversidad y consideraciones multiculturales, la incorporación de intervención temprana, además de la accesibilidad y el financiamiento de educación especial. La interpretación de los resultados busca describir las similitudes y diferencias en la implementación de políticas de educación especial inclusiva dentro de España y los Estados Unidos con referencia a las políticas educativas supranacionales sobre educación inclusiva.

\section{Abstract}

Open global communication provides access to a shared network of evidence and experience regarding policies and practices on an immense scope of issues. The cooperation between individuals, agencies, non-governmental organizations, countries, and international organizations creates a perpetual, dynamic collection of information that serves as a global bank of knowledge. Supranational education policies contribute consolidated guidelines and ideas for countries to adapt into national policy. In the field of education, inclusion of children with special educational needs has evolved into an important global discussion and is established as an essential component of education policy. This paper aims to analyze the alignment of supranational education policy regarding inclusive education of students with special educational needs with the national education policies of Spain and the United States, in order to evaluate the variability between policies as well as enable recommendations for future actions to continue the improvement of inclusive education. Supranational education policy of international organizations such as the United Nations, United Nations Educational, Scientific, and Cultural Organization (UNESCO), Organization for Economic Co-operation and Development (OECD), and United Nations (UN) are incorporated. National educational policies focus on the Individuals with Disabilities Education Act (IDEA) in the U.S., and the Ley Orgánica 2/2006, de 3 de mayo, de Educación (LOE) in Spain. This study compares and evaluates the national education policies of Spain and the United States regarding the following aspects: inclusive education laws, guidelines and processes, inclusive placement settings, interdisciplinary service provision, diversity and multicultural considerations, the enactment of early intervention services, in addition to accessibility and financing of special education. The interpretation of these results seeks to describe the similarities and differences in the implementation of inclusive special education policies within Spain and the U.S. with reference to supranational education policies on inclusive education.

Palabras clave: Política Educativa Supranacional, Inclusión, Educación Inclusiva, Educación Especial, España, Estados Unidos.

Keywords: Supranational Education Policy, Inclusion, Inclusive Education, Special Education, Spain, United States. 


\section{INTRODUCCIÓN}

Un tema que ha evolucionado a lo largo de las últimas siete décadas es la inclusión educativa de las personas con discapacidad. Existe un alcance amplio de Política Educativa Supranacional (PES) que aporta pautas y recomendaciones para que los países se adapten a la política nacional. La formulación de la política nacional tiene en cuenta las diversas necesidades de la población, así como las implicaciones históricas y culturales, a fin de mejorar las estrategias y los procedimientos dados los objetivos del país. Las reformas de políticas se derivan de los movimientos hacia las mejores prácticas a medida que se adquiere la evidencia. Inicialmente, el enfoque de la investigación era definir y promover la idea de educación inclusiva. Actualmente, el objetivo ya no es responder a las cuestiones de «qué» y «por qué» sino de "cómo» (EASNIE, 2017b). Posteriormente, hay una continuación de los avances nacionales e internacional es en la investigación y las prácticas hacia la implementación de la inclusión educativa.

Este estudio apunta a utilizar la política educativa supranacional sobre la educación inclusiva de estudiantes con discapacidades para desarrollar una colección de indicadores, con los cuales comparar laspolíticasnacionalesdeeducacióndeEspañaylosEstadosUnidos(EEUU), conlafinalidaddeevaluar la variabilidad entre las políticas y posibilitar reflexiones y recomendaciones para futuras acciones para mejorar la educación inclusiva al nivel nacional. Los indicadores están derivados de las políticas educativas supranacionales con referencia a educación especial e inclusiva, de organizaciones internacionales como la Organización de las Naciones Unidas para la Educación, la Ciencia y la Cultura (UNESCO), la Organización para la Cooperación y el Desarrollo Económicos (OCDE), y la Organización de Naciones Unidas (ONU). La comparación se centra en las políticas nacionales de España y EEUU sobre educación especial e inclusiva, dentro de los marcos jurídicos y teóricos. A partir del análisis de la yuxtaposición de las políticas nacionales, la conclusión fórmula una discusión sobre la alineación con PES y reflexiones.

\section{POLÍTICA EDUCATIVA SUPRANACIONAL}

La Política Educativa Supranacional (PES) se sitúa dentro de las relaciones mutuas de política educativa, educación comparada, y educación internacional (Valle, 2012). Existe una cantidad inmensa de políticas supranacionales que forman referencias globales a los estándares y derechos compartidos por todas las personas, acordados por los países signatarios. Este artículo anota cinco de las PES más recientes y comprensivas sobre inclusión educativa de personas con discapacidad:

- UNESCO Declaración de Salamanca (1994)

- OECD Education Policy Analysis: Chapter 1 (2003)

- UN Convention on the Rights of Persons with Disabilities (2006)

- UNESCO Policy Guidelines on Inclusion in Education (2009)

- UNESCO Incheon Declaration: Education 2030 (2015)

Estos documentos fueron revisados con el propósito de desarrollar los indicadores para comparar las políticas de España y EEUU. Estas «soft policies» vinculan los conceptos de inclusión educativa a las prácticas pertinentes y recomendadas a través de diez áreas impactantes de educación inclusiva. Se destacan factores principales que se supone que orientan las políticas educativas nacionales hacia la institución de la inclusión educativa al nivel nacional, y posteriormente, local. 
Figura 1. Cuadro comparativo de educación inclusiva: Categorías, Parámetros, e Indicadores. Elaboración personal a partir de las fuentes citadas en Anexo 3.

\begin{tabular}{|c|c|c|}
\hline Categorías & Parámetros & Indicadores \\
\hline \multirow{6}{*}{ Inversión } & \multirow{2}{*}{ Inversión general en educación } & $\%$ de Gasto Gubernamental \\
\hline & & $\%$ de Gasto Público \\
\hline & Inversión en educación especial & \% Gasto en educación apropiado a educación especial \\
\hline & Inversión por cada estudiante & $\begin{array}{l}\text { \$ Gasto por estudiante } \\
\text { (dólar estadounidense) }\end{array}$ \\
\hline & \multirow{2}{*}{ Población con necesidades especiales } & $\%$ de niños con necesidades especiales \\
\hline & & $\%$ registrado en marco inclusiva \\
\hline \multirow{4}{*}{$\begin{array}{l}\text { Accesibilidad y } \\
\text { Recursos }\end{array}$} & \multirow{3}{*}{ Financiación } & Políticas de financiación \\
\hline & & $\begin{array}{l}\text { Recursos adecuados y equilibrados en } \\
\text { escuelas públicas como privadas }\end{array}$ \\
\hline & & Acceso gratis \\
\hline & Igualdad & $\begin{array}{l}\text { Regulaciones que garantizar equidad y prevenir } \\
\text { discriminación y desigualdad }\end{array}$ \\
\hline \multirow{4}{*}{$\begin{array}{l}\text { Colaboración y } \\
\text { Responsabilidad }\end{array}$} & Familiar & $\begin{array}{l}\text { Participación del individuo y familias en } \\
\text { toma de decisiones, recursos y apoyo }\end{array}$ \\
\hline & Comunitaria & $\begin{array}{l}\text { Coordinación comunitaria y integración social a } \\
\text { través de las esferas de salud, laboral, programas de } \\
\text { rehabilitación e infraestructura integrada }\end{array}$ \\
\hline & \multirow{2}{*}{ Gobierno } & $\begin{array}{l}\text { Asignación de recursos humanos, institucionales, } \\
\text { logísticos, materiales, financieros, etc. }\end{array}$ \\
\hline & & Políticas / leyes de inclusión educativa \\
\hline $\begin{array}{l}\text { Consideraciones } \\
\text { Multiculturales }\end{array}$ & $\begin{array}{l}\text { Reconocimiento de grupos vulnerables } \\
\text { en políticas educativas }\end{array}$ & $\begin{array}{l}\text { Políticas que respondan a la diversidad y las nece- } \\
\text { sidades como discapacidad, diversidad lingüística, } \\
\text { personas indígenas, minorías, y los pobres. }\end{array}$ \\
\hline \multirow{2}{*}{$\begin{array}{l}\text { Formación del } \\
\text { Profesorado }\end{array}$} & \multirow{2}{*}{$\begin{array}{l}\text { Preparación para trabajar con estudian- } \\
\text { tes con discapacidades }\end{array}$} & Formación inicial en universidad \\
\hline & & Formación colaborativo en el servicio \\
\hline \multirow{4}{*}{$\begin{array}{l}\text { Procedimientos } \\
\text { Preliminares }\end{array}$} & \multirow{2}{*}{ Requisitos iniciales } & Requisitos para el proceso de referencia \\
\hline & & Requisitos para el proceso de evaluación \\
\hline & \multirow{2}{*}{ Estándares de elegibilidad } & $\begin{array}{c}\text { Definición de discapacidad o } \\
\text { necesidades educativas especiales }\end{array}$ \\
\hline & & $\begin{array}{l}\text { Criterios de elegibilidad claros para determinar si } \\
\text { una necesidad especial está presente }\end{array}$ \\
\hline
\end{tabular}




\begin{tabular}{|c|c|c|}
\hline \multirow{2}{*}{ Ubicaciones Inclusivas } & \multirow{2}{*}{$\begin{array}{c}\text { Políticas establecidas para la inclusión } \\
\text { educativa - mainstreaming }\end{array}$} & Participación requerida en escuelas ordinarias \\
\hline & & $\begin{array}{l}\text { Niveles de inclusión relacionadas con la } \\
\text { gravedad; individualizada }\end{array}$ \\
\hline \multirow{3}{*}{ Provisión de Servicios } & Enfoque en equipo interdisciplinario & $\begin{array}{l}\text { Incluye servicios de desarrollo, psicológicos, } \\
\text { físicos, ocupacionales, de comunicación y } \\
\text { auditivos / visuales en la escuela }\end{array}$ \\
\hline & \multirow{2}{*}{ Planificación e Implementación } & $\begin{array}{c}\text { Pautas de documentación formal y concisa } \\
\text { para la implementación de servicios individualizada }\end{array}$ \\
\hline & & $\begin{array}{l}\text { Ayudas técnicas que facilitan la comunicación, la } \\
\text { movilidad, y el aprendizaje (apoyo que requieran) }\end{array}$ \\
\hline \multirow{3}{*}{$\begin{array}{l}\text { AtenciónTemprana } \\
\text { / EducaciónInfantil }\end{array}$} & \multirow{2}{*}{ Accesibilidad } & Pronto identificación de edades 0-6 años \\
\hline & & Accesibilidad a servicios regulada por ley \\
\hline & Ambiente & $\begin{array}{l}\text { Servicios provistos en entorno natural } \\
\text { con colaboración familiar y comunitaria }\end{array}$ \\
\hline Educación de Adultos & $\begin{array}{l}\text { Extensión de educación inclusiva a adul- } \\
\text { tos con discapacidad }\end{array}$ & $\begin{array}{l}\text { Regulación de programas de transición, } \\
\text { formación profesional, e inserción laboral }\end{array}$ \\
\hline
\end{tabular}

Fuente: elaboración propia.

\section{POLÍTICAS EDUCATIVAS NACIONALES}

Todos los indicadores fueron analizados y valorados mediante la investigación de políticas educativas nacionales sobre la inclusión de personas con discapacidad en España y EEUU. Aunque los resultados completos aparecen en la tabla comparativa del próximo capítulo, algunos de los indicadores más pertinentes están extendidos en las secciones siguientes, debido a la necesidad de utilizar el espacio con eficiencia.

\subsection{Inversión}

Como punto de referencia, las inversiones designadas por el gobierno reflejan el nivel de interés y la prioridad otorgada a la educación. En las estadísticas de España y EEUU se obtuvieron de varias fuentes y organismos internacionales con respecto al gasto en educación en general, seguidas por el área específica de educación especial. El porcentaje de niños con discapacidad, el porcentaje de estudiantes con Necesidades Educativas Especiales (NEE) educados en un marco inclusivo, la edad media de la población, y el porcentaje de la población en edad escolar se detallan más adelante en este informe.

\subsubsection{España}

- 6.0\% del gasto público es para educación (OECD, 2018b).

- 3.3\% del gasto público en educación es para «otros servicios educativos» los cuales incluyen educación especial (Ministerio de Educación, Cultura y Deporte [MECD], 2016).

- $2.84 \%$ de niños registrado en la escuela tiene una discapacidad (European Agency for Special Needs and Inclusive Education [EASNIE], 2017a).

- $77 \%$ de niños con NEE están en mainstream educación inclusiva a menos de $80 \%$ del tiempo (EASNIE, 2017a). 
Cabe destacar que menos de un cuarto de la población está en edad escolar, además de un ratio bajo de niños con discapacidad, los que posiblemente explican el presupuesto educativo relativamente bajo, debido a la disminución del número de personas atendidas en este sector. España coloca la mayoría de estudiantes con discapacidad en mainstream educación inclusiva como mínimo 80\% del tiempo.

\subsubsection{Estados Unidos}

- 8.3\% del gasto público es para educación (OECD, 2018b)

- 18.9\% del presupuesto federal de educación se asigna a la educación especial; Asignación de \$13 mil millones a educación especial dividida por $\$ 64.9$ mil millones de presupuesto total de educación (U.S. Department of Education [DOE], 2017a,b).

- 13\% de niños (3-21 años) registrado en escuelas públicas tiene una discapacidad (National Center for Education Statistics [NCES], 2017).

- $62.2 \%$ de niños (6-21 años) con NEE están en mainstream educación inclusiva a menos de $80 \%$ del tiempo (NCES, 2016).

Cabe destacar que hay una proporción relativamente alta de niños con discapacidad, lo que posiblemente explica el presupuesto educativo alto. EEUU colocan la mayoría de estudiantes con discapacidad en «mainstream» educación inclusiva como mínimo el $80 \%$ del tiempo.

\subsection{Accesibilidad y Recursos}

El acceso a una educación inclusiva debe ser gratis, además de que el país tiene otras políticas colocadas sobre la financiación de educación. Recursos deben ser adecuados y equilibrados en escuelas públicas como privadas. Conjuntamente, las regulaciones que garantizan la equidad y previenen la discriminación promueven la igualdad educativa.

\subsubsection{España}

La Constitución Española (CE), artículo 27, documenta que «la enseñanza básica es obligatoria y gratuita» (CE, 2018, p.6), seguido por la Ley Orgánica 2/2006, de 3 de mayo, de Educación [LOE] (Agencia Estatal Boletín Oficial del Estado [BOE], 2006), que justifica la norma y ratifica la ley de educación gratuita. Todas las leyes españolas incorporan la igualdad en políticas contra la discriminación de personas con discapacidad (BOE, 1995; BOE, 2006; BOE, 2013).

\subsubsection{Estados Unidos}

La Elementary and Secondary Education Act (ESEA) de 1965, reautorizado en 2015 por la Every Student Succeeds Act (ESSA), requiere una educación pública gratuita desde la escuela primaria hasta el fin de secundaria, mientras la IDEA normaliza una Free Appropriate Public Education (FAPE) para los alumnos con discapacidad en particular, de edades 3-21 años (FAPE, 2018). Las normas de Discrimination prohibited (2018) declaran la prohibición de acciones específicas de discriminación basada en raza, color, nacionalidad o discapacidad, exclusivamente dentro de programas de educación y empleo que reciben ayuda financiera federal (DOE, 2008).

\subsection{Colaboración y Responsabilidad}

La educación inclusiva requiere la participación activa del individuo y las familias en la toma de decisiones. El sistema promueve integración social a través de la conexión con la comunidad en esferas de salud, laboral, programas de rehabilitación e infraestructura integrada. Además de políticas que 
explícitamente exige la inclusión educativa, el gobierno debe incluir políticas que asignan recursos eficaces - recursos humanos, institucionales, logísticos, materiales, financieros, etc.

\subsubsection{España}

La participación de los padres está regulada por el Real Decreto 696/1995, de 28 de abril, de ordenación de la educación de los alumnos con necesidades educativas especiales (BOE, 1995) y la LOE (2006), así como mencionada en el Plan de inclusión del alumnado con necesidades educativas especiales [PINEE] (Ministerio de Educación, 2011), todos los cuales promueven la comunicación y la toma de decisiones colaborativas. A nivel comunitario, el Real Decreto (BOE, 1995) y la LOE (2006) respaldan la colaboración entre las administraciones educativas y otras instituciones o asociaciones, entidades públicas o privadas sin ánimo de lucro, pero el PINEE (Ministerio de Educación, 2011) da ejemplos como las administraciones de salud y servicios sociales.

\subsubsection{Estados Unidos}

Parent and family engagement (2015) demanda que la agencia educativa local conceptualiza políticas que involucran a los padres y familias en programas, actividades, y procedimientos educativos, además de la participación de padres en las reuniones y la toma de decisiones sobre los niños con discapacidad (Parent participation, 2018). Desde la perspectiva de colaboración comunitaria, la ESEA (2015) y el Department of Education (2008) hace referencias múltiples a la cooperación de la agencia educativa con varias organizaciones, escuelas, empresas, servicios y líderes de la comunidad.

\subsection{Consideraciones Multiculturales}

Para reconocer la variedad de la población educativa, hay que realizar políticas que responden a la diversidad y las necesidades como discapacidad, diversidad lingüística, personas indígenas, minorías y los pobres.

\subsubsection{España}

La LOE (2006) destaca la diversidad cultural, lingüística, social, de género y de aptitudes, y por eso implementan políticas para resolver conflictos y evitar la discriminación en general. El Plan nacional de acción para la inclusión social del Reino de España [PNAIN] (Ministerio de Sanidad, Servicios Sociales e Igualdad, 2013) presenta objetivos dentro del ámbito de educación, que se manifiestan en actuaciones organizadas por cada grupo vulnerable.

\subsubsection{Estados Unidos}

La ESEA (2015) e IDEA (2004) reconoce la diversidad lingüística, cultural, geográfica, y económica, con el objetivo de romper las barreras a una educación equitativa para los colectivos en riesgo de exclusión como los pobres, minorías, rurales, abandonados, delincuentes y personas sin hogar o con discapacidad.

\subsection{Formación del Profesorado}

Las políticas educativas sobre la formación del profesorado influyen en el éxito de inclusión, iniciando al nivel universitario durante la formación inicial y continuando a lo largo del servicio docente para prepararlos para trabajar con estudiantes con discapacidades en la aula. 


\subsubsection{España}

Los marcos jurídicos y teóricos de España exhiben la importancia de la formación del profesorado para el trabajo con los alumnos con discapacidades. EI Real Decreto (BOE, 1995) y la LOE (2006) especifican las regulaciones para la formación inicial y permanente de docentes. Una cantidad de «soft policy» refuerzan las mismas aportaciones como las actuaciones del PINEE (Ministerio de Educación, 2011), orientadas a la formación inicial, permanente, especialistas, equipos directivos y servicios de orientación educativa para la detección temprana.

\subsubsection{Estados Unidos}

Use of funds (2004) proporciona fondos para la formación profesional continua de docentes y personal sobre la instrucción, intervención, planificación y el desarrollo de los alumnos con discapacidades.Personnel qualifications (2018) puntualiza las calificaciones necesarias del personal docente y de servicios relacionados a través de la formación inicial y continua para trabajar con individuos con discapacidad.

\subsection{ProcedimientosPreliminares}

Hay dos componentes antes de la determinación de elegibilidad para una necesidad educativa especial (NEE), la referencia y la evaluación, para las cuales debe haber un proceso y requisitos en su puesto. Para establecer una determinación de elegibilidad para servicios de necesidades especiales, se debe desarrollar una definición de NEE, además de criterios para determinar si hay algún tipo de NEE presente.

\subsubsection{España}

No aparece una definición de necesidades educativas especiales concreta ni consistente en las leyes educativas españolas. La única definición de NEE es de la LOE, artículo 73, que «se entiende por alumnado que presenta necesidades educativas especiales, aquel que requiera, por un periodo de su escolarización o a lo largo de toda ella, determinados apoyos y atenciones educativas específicas derivadas de discapacidad o trastornos graves de conducta» (BOE, 2006, p.54). En términos de elegibilidad para servicios especiales, Real Decreto (BOE, 1995) cita las discapacidades psíquica, motora y sensorial con frecuencia, entre otras mencionadas a lo largo del decreto como trastornos graves de conducta y personalidad, discapacidades de comunicación y lenguaje, visuales, auditivas, mentales y autismo. Eurydice (2015) proporciona categorías de necesidades educativas especiales en España: trastornos físicos, sensoriales, motores y de conducta, trastorno por déficit de atención e hiperactividad (TDAH) y dificultades específicas de aprendizaje. No se incluye ninguna elaboración sobre entidades específicas de estas categorías.

\subsubsection{Estados Unidos}

La elegibilidad se determina por la interpretación de la información evaluativa (Determination of eligibility, 2018) para diagnosticar el niño con una discapacidad según la definición en la ley. Child with a disability (2018) delimita las 14 categorías de elegibilidad con criterios de calificación detallados para determinar la presencia de una discapacidad y la necesidad educativa de los servicios para abordarla. Un estudiante no es elegible para recibir servicios si muestra un dominio limitado del inglés o si hay una falta de instrucción (Determination of eligibility, 2018).

\subsection{Ubicaciones Inclusivas}

Mainstreaming requiere la ubicación de niños con necesidades especiales en el mismo entorno educativo que sus compañeros sin necesidades especiales, así como el acceso al currículo normal. La 
colocación se refiere a las configuraciones en las cuales se lleva a cabo la educación. Normalmente hay dos opciones distintas: escuela regular o escuela especial. Afortunadamente, con el inicio del movimiento de inclusión, se está creando un espectro de opciones de ubicación que estará disponible.

\subsubsection{España}

El gobierno español requiere la inclusión educativa y solo permitirá la escolarización en centros de educación especial cuando sus necesidades no puedan ser atendidas en el marco de atención a la diversidad de los centros y programas ordinarios (BOE, 1995; BOE, 2006, Ministerio de Educación, 2011). España destaca tres escenarios de colocación: centros ordinarios, hospitalarios o de rehabilitación, y centros de educación especial, los cuales tienen criterios y reglas de organización (BOE,1995).

\subsubsection{Estados Unidos}

La IDEA instituye un requisito para la colocación de estudiantes con discapacidad en el Least Restrictive Environment (LRE, 2018), que permite un continuo de ubicaciones alternativas con cinco opciones para entornos educativos desde los menos restrictivos a los más restrictivos: clases regulares, clases especiales, escuelas especiales, instrucción en el hogar o instrucción en hospitales e instituciones. Sin embargo, debe haber una razón debido a la naturaleza o la gravedad de la discapacidad para justificar la remoción de los estudiantes de la educación regular (LRE, 2018).

\subsection{Provisión de Servicios}

La colaboración de un equipo interdisciplinario es imprescindible para un proceso integral y la provisión de servicios. Los miembros del equipo varían según las necesidades del niño, pero deben incorporarse en las políticas de planificación e implementación de los servicios. La provisión de servicios de educación especial debe ser estructurada por un plan específico, documentado formalmente, para guiar la implementación, además se permite la revisión. Facilita el desarrollo de objetivos y métodos para favorecer la provisión adecuada de adaptaciones y ayudas técnicas para abordar las NEE.

\subsubsection{España}

El Real Decreto (BOE, 1995) es la única ley que destaca la posiciones del equipo de orientación educativa y las especialidades como pedagogía terapéutica, educación especial, psicología, audición y lenguaje. Además, especialistas como psicólogos infantiles, logopedas, fisioterapeutas,psicopedagogos, trabajadores sociales e intérpretes del lenguaje de signos, también el rol del tutor, están indicados desde otras fuentes, pero la elaboración de las entidades específicas de los servicios es limitada (Ministerio de Educación, 2011; Universidad Internacional de Valencia, 2014).

\subsubsection{Estados Unidos}

El equipo del Individualized Education Program (IEP) debe ser multidisciplinario de acuerdo con las necesidades del caso, y la IDEA incluye los siguientes actores con responsabilidades elaboradas para cada puesto: padres, profesor de educación regular y de educación especial, un representante de la agencia, especialidades de terapia (logopeda, audiología, ocupacional y física), psicólogo, consejero, asistente social, servicios de salud, especialista en desarrollo, etc. (Related services, 2018; IEP, 2018). Programas como Response to Intervention (RTI) y Positive Behavior Intervention and Support (PBIS) facilitan la intervención inclusiva (TASH, s.f.). 


\subsection{Atención Temprana / Educación Infantil}

La educación comienza al nacer y es imprescindible en los años formativos, edades 0-6, identificar temprano cualquier necesidad especial y proveer acceso a servicios, los cuales se proporcionan en el entorno natural con colaboración familiar y comunitaria.

\subsubsection{España}

Las leyes (BOE 1995; BOE 2006, BOE, 2013) instituyen el requisito de la detección temprana de necesidades educativas especiales con referencia a cualquier nivel escolar, pero el PINEE (Ministerio de Educación, 2011) identifica una necesidad de potenciar la evaluación y detección temprana en las edades de 0-6 años. La implementación de la educación infantil está regulada por artículos 12-15 de la LOE (2006), que prescribe las edades, los objetivos, la gratuidad, la evaluación y los servicios, además del Real Decreto que asigna «particularmente en la que se lleve a cabo en edades anteriores a la escolarización, el Ministerio de Educación y Ciencia propiciará de manera especial la colaboración de los padres o tutores de los niños y niñas, los cuales podrán recibir preparación a tal fin ofrecida por los servicios correspondientes» (BOE, 1995, p.8).

\subsubsection{Estados Unidos}

La IDEA (2004) y el Policy statement on inclusion of children with disabilities in early childhood programs (U.S. Department of Heath and Human Services \& U.S. Department of Education, 2015) reconocen la importancia de identificación temprana de niños menores de cinco años con discapacidad y su inclusión en la sociedad a través de la conciencia pública, formación docente y paterna y servicios relacionados.

\subsection{Educación de Adultos}

La regulación de los programas de transición y la formación profesional forma una parte significativa de la trayectoria desde inclusión educativa hasta inclusión laboral, la que facilita el éxito continuo de personas con discapacidades.

\subsubsection{España}

Todas las leyes (BOE 1995; BOE 2006, BOE, 2013) y el PINEE (Ministerio de Educación, 2011) desarrollan la etapa más allá de la educación básica y obligatoria para el alumnado con discapacidades. Hay reglamentos y normas para la transición a la vida adulta, la formación profesional e inserción laboral, además de educación para adultos que tiene en cuenta la presencia de necesidades educativas especiales.

\subsubsection{Estados Unidos}

Transition Services (2004) abarca el proceso del movimiento de estudiantes con discapacidad desde escuela secundaria a formación profesional, educación para adultos, inserción laboral, y vida independiente. La Office of Special Education and Rehabilitative Services (OSERS) es responsable para la rehabilitación vocacional de personas con discapacidad (DOE, 2008).

\section{ANÁLISIS COMPARATIVO}

En primer lugar, para visualizar la yuxtaposición de los hallazgos de las políticas educativas nacionales de España y EEUU, se manifiesta un cuadro completo con los resultados de los indicadores por país. 
Figura 2. Cuadro comparativo de educación inclusiva. Resultados por país: España y los Estados Unidos. Elaboración personal a partir de la información citada en capítulo 2

\begin{tabular}{|c|c|c|c|c|}
\hline Categorías & Parámetros & Indicadores & España & EEUU \\
\hline \multirow{6}{*}{ Inversión } & \multirow{2}{*}{$\begin{array}{l}\text { Inversión general } \\
\text { en educación }\end{array}$} & $\%$ de Gasto Gubernamental & $9.593 \%$ & $14.548 \%$ \\
\hline & & $\%$ de Gasto Público & $6.0 \%$ & $8.3 \%$ \\
\hline & $\begin{array}{l}\text { Inversión en } \\
\text { educación especial }\end{array}$ & $\begin{array}{c}\text { \% Gasto en educación apropiado a } \\
\text { educación especial }\end{array}$ & $3.3 \%$ & $18.9 \%$ \\
\hline & $\begin{array}{l}\text { Inversión por } \\
\text { cada estudiante }\end{array}$ & $\begin{array}{c}\text { \$ Gasto por estudiante } \\
\text { (dólar estadounidense - USD) }\end{array}$ & $\begin{array}{l}3,000 \\
\text { USD }\end{array}$ & $\begin{array}{l}12,200 \\
\text { USD }\end{array}$ \\
\hline & \multirow{2}{*}{$\begin{array}{l}\text { Población con } \\
\text { necesidades } \\
\text { especiales }\end{array}$} & $\begin{array}{l}\% \text { de niños (registrados en escuela) con } \\
\text { necesidades educativas especiales }\end{array}$ & $2.84 \%$ & $13 \%$ \\
\hline & & $\begin{array}{l}\% \text { registrado en marco inclusiva } \\
\text { (a menos de } 80 \% \text { en mainstream) }\end{array}$ & $77 \%$ & $62.2 \%$ \\
\hline \multirow{4}{*}{$\begin{array}{l}\text { Accesibilidad y } \\
\text { Recursos }\end{array}$} & \multirow{3}{*}{ Financiación } & Políticas de financiación & General & Sí \\
\hline & & $\begin{array}{l}\text { Recursos adecuados y equilibrados en } \\
\text { escuelas públicas como privadas }\end{array}$ & Sí & Sí \\
\hline & & Acceso gratis & Sí & Sí \\
\hline & Igualdad & $\begin{array}{l}\text { Regulaciones que garantizar equidad y } \\
\text { prevenir discriminación y desigualdad }\end{array}$ & Sí & Sí \\
\hline \multirow{4}{*}{$\begin{array}{l}\text { Colaboración y } \\
\text { Responsabilidad }\end{array}$} & Familiar & $\begin{array}{l}\text { Participación del individuo y familias en toma } \\
\text { de decisiones, recursos y apoyo }\end{array}$ & Sí & Sí \\
\hline & Comunitaria & $\begin{array}{l}\text { Coordinación comunitaria y integración } \\
\text { social a través de las esferas de salud, } \\
\text { laboral, programas de rehabilitación e } \\
\text { infraestructura integrada }\end{array}$ & Sí & Sí \\
\hline & \multirow[t]{2}{*}{ Gobierno } & $\begin{array}{c}\text { Asignación de recursos humanos, } \\
\text { institucionales, logísticos, } \\
\text { materiales, financieros, etc. }\end{array}$ & Sí & Sí \\
\hline & & Políticas / leyes de inclusión educativa & Sí & No \\
\hline $\begin{array}{l}\text { Consideraciones } \\
\text { Multiculturales }\end{array}$ & $\begin{array}{l}\text { Reconocimiento de } \\
\text { grupos vulnerables }\end{array}$ & $\begin{array}{c}\text { Políticas que respondan a la diversidad y las } \\
\text { necesidades como discapacidad, } \\
\text { diversidad lingüística, personas } \\
\text { indígenas, minorías,y los pobres. }\end{array}$ & Sí & Sí \\
\hline \multirow[b]{2}{*}{$\begin{array}{l}\text { Formación del } \\
\text { Profesorado }\end{array}$} & \multirow{2}{*}{$\begin{array}{l}\text { Preparaciónpara } \\
\text { trabajar con } \\
\text { estudiantes con } \\
\text { discapacidades }\end{array}$} & Formación inicial en universidad & Sí & Sí \\
\hline & & Formación colaborativo en el servicio & Sí & Sí \\
\hline
\end{tabular}




\begin{tabular}{|c|c|c|c|c|}
\hline \multirow{4}{*}{$\begin{array}{l}\text { Procedimientos } \\
\text { Preliminares }\end{array}$} & \multirow{2}{*}{ Requisitos iniciales } & Requisitos para el proceso de referencia & No & Sí \\
\hline & & Requisitos para el proceso de evaluación & General & Sí \\
\hline & \multirow[b]{2}{*}{$\begin{array}{l}\text { Estándares de } \\
\text { elegibilidad }\end{array}$} & $\begin{array}{c}\text { Definición de discapacidad o necesidades } \\
\text { educativas especiales }\end{array}$ & General & Sí \\
\hline & & $\begin{array}{l}\text { Criterios de elegibilidad claros para } \\
\text { determinar si una necesidad } \\
\text { especial está presente }\end{array}$ & General & Sí \\
\hline \multirow{2}{*}{$\begin{array}{l}\text { Ubicaciones } \\
\text { Inclusivas }\end{array}$} & \multirow{2}{*}{$\begin{array}{c}\text { Políticas estableci- } \\
\text { das para la } \\
\text { inclusión educativa }\end{array}$} & $\begin{array}{l}\text { Participación requerida en escuelas } \\
\text { ordinarias - mainstreaming }\end{array}$ & Sí & Sí \\
\hline & & $\begin{array}{c}\text { Niveles de inclusión relacionadas con la } \\
\text { gravedad; individualizada }\end{array}$ & Sí & Sí \\
\hline \multirow{3}{*}{$\begin{array}{l}\text { Provisión de } \\
\text { Servicios }\end{array}$} & $\begin{array}{l}\text { Enfoque en equipo } \\
\text { interdisciplinario }\end{array}$ & $\begin{array}{c}\text { Incluye servicios de desarrollo, psicológicos, } \\
\text { físicos, ocupacionales, de comunicación y } \\
\text { auditivos / visuales }\end{array}$ & Sí & Sí \\
\hline & \multirow{2}{*}{$\begin{array}{l}\text { Planificación e } \\
\text { Implementación }\end{array}$} & $\begin{array}{l}\text { Pautas de documentación formal y } \\
\text { concisa para la implementación de } \\
\text { servicios individualizada }\end{array}$ & No & Sí \\
\hline & & $\begin{array}{c}\text { Ayudas técnicas que facilitan la comunicación, } \\
\text { la movilidad, y el aprendizaje } \\
\text { (apoyo que requieran) }\end{array}$ & Sí & Sí \\
\hline \multirow{3}{*}{$\begin{array}{l}\text { Atención Temprana } \\
\text { / Educación Infantil }\end{array}$} & \multirow{2}{*}{ Accesibilidad } & Pronto identificación de edades 0-6 años & Sí & Sí \\
\hline & & Accesibilidad a servicios regulada por ley & Sí & Sí \\
\hline & Ambiente & $\begin{array}{l}\text { Servicios provistos en entorno natural con } \\
\text { colaboración familiar y comunitaria }\end{array}$ & Sí & Sí \\
\hline $\begin{array}{l}\text { Educación de } \\
\text { Adultos }\end{array}$ & $\begin{array}{c}\text { Extensión de } \\
\text { educación } \\
\text { inclusiva a adultos- } \\
\text { con discapacidad }\end{array}$ & $\begin{array}{l}\text { Regulación de programas de transición, } \\
\text { formación profesional, e inserción laboral }\end{array}$ & sí & Sí \\
\hline
\end{tabular}

Fuente: elaboración propia.

Al principio, EEUU gasta una cantidad significativa más que España en educación regular y especial,pero tiene una población con discapacidad más alta. Con relación a la inclusión, España reporta un alto porcentaje $(77 \%)$, en comparación a EEUU $(62.2 \%)$, de niños con discapacidad educados en la clase regular menos del $80 \%$ del tiempo. Considerando las otras nueve categorías, la mayoría (17) de las respuestas a los 24 indicadores restantes en ambos países se marcaron como sí, que se convergen en la alineación de las políticas nacionales con los indicadores de PES. Un indicador de EEUU y dos de España fueron marcados como no y cuatro indicadores de España marcados como general (Figura 2). La consideración de estas divergencias se manifiesta en unos hallazgos claves que corresponden a la descentralización de los dos sistemas nacionales. Por parte de España, la falta de leyes financieras, conjuntamente con la ambigüedad en su lenguaje jurídico sobre la definición, evaluación, elegibilidad y el plan de servicios, exhiben una gran autonomía de las Comunidades Autónomas (CCAA) para financiar, identificar, definir, evaluar e intervenir en casos de NEE. Sin embargo, los recursos reflexivos sobre el sistema educativo español amplían los objetivos, modelos y procedimientos mientras que la legislación se alinea generalmente con PES. Por parte de EEUU, existe una falta de generalización de los conceptos que vienen de PES como la inclusión y la prohibición de discriminación más allá de los programas que reciben fondos federales. El indicador sobre Políticas de inclusión educativa es el único indicar que no tiene EEUU debido a la 
IDEA que nunca refiere a la palabra inclusión, solo el LRE, que enlaza la inclusión, pero no explícitamente. Aunque EEUU tiene un sistema descentralizado también, se concretan políticas educativas sobre personas con discapacidad al nivel federal, requiriendo el cumplimiento con las políticas por parte de los estados para obtener financiamiento federal. Estos hallazgos indican que la inclusión es una prioridad internacional, a pesar de la variabilidad entre la autonomía y las políticas nacionales, hacia la mejora continua en estos sistemas escolares.

\section{DISCUSIÓN Y CONCLUSIONES}

Con respeto a este estudio, no pretende ser comprensivo o profundo en términos de la complejidad y consideración de todos los factores que influyen en las políticas educativas nacionales. Es imprescindible comprender el concepto de inclusión no solo dentro de actuaciones políticas, sino considerar la práctica y el esfuerzo social igualmente. Las políticas no sirven para nada si no están acompañadas por el compartir del conocimiento y la colaboración de los miembros de la sociedad para establecer la implementación de inclusión educativa a nivel local. España y EEUU ejemplifican la participación en un movimiento global hacia inclusión a través de la alineación con PES. Los dos pueden extender su alcance de inclusión con políticas más amplias sobre inclusión y anti-discriminación por parte de EEUU, y con políticas más concentradas en España sobre la definición, evaluación y planificación para mantener un enfoque sistematizado entre CCAA, todo con la finalidad de un reconocimiento global de la promoción de equidad en educación inclusiva. Cabe destacar la difusión de sistemas descentralizados a partir de la Declaración de Salamanca (1994), que promueve la inclusión educativa desde las agencias educativas locales. El eje es la implementación de educación inclusiva, que no es necesariamente solo top-down o bottom-up, sino que viene de la consideración de PES y la interacción de ella con las políticas educativas nacionales, aplicadas por agencias y escuelas locales que participan en el desarrollo de buenas prácticas y retroalimentación para mejorar políticas además de prácticas.

\section{REFERENCIAS BIBLIOGRÁFICAS}

Agencia Estatal Boletín Oficial del Estado. (1995). Real Decreto 696/1995, de 28 de abril, de ordenación de la educación de los alumnos con necesidades educativas especiales. Recuperado de https://www.boe.es/buscar/pdf/1995/ BOE-A-1995-13290-consolidado.pdf.

Agencia Estatal Boletín Oficial del Estado. (2006). Ley Orgánica 2/2006, de 3 de mayo, de Educación. Recuperado de https://www.boe.es/buscar/pdf/2006/BOE-A-2006-7899-consolidado.pdf.

Agencia Estatal Boletín Oficial del Estado. (2013). Ley Orgánica 8/2013, de 9 de diciembre, para la mejora de la calidad educativa. Recuperado de http://www.boe.es/boe/dias/2013/12/10/pdfs/BOE-A-2013-12886.pdf.

Agencia Estatal Boletín Oficial del Estado. (2014). Real Decreto 126/2014, de 28 de febrero, por el que se establece el currículo básico de la Educación Primaria. Recuperado de http://www.boe.es/boe/dias/2014/03/01/pdfs/BOE-A-2014-2222.pdf.

Aragall, F. (2010). La accesibilidad en los centros educativos. Recuperado de http://www.mecd.gob.es/educacion-mecd/ $\mathrm{dms} / \mathrm{mecd} /$ educacion-mecd/areas-educacion/sistema-educativo/educacion-inclusiva/necesidad-apoyo-educativo/ alumnado-necesidades-especiales/2010-accesibilidad-centros-educativos-mayo-pdf.pdf.

Central Intelligence Agency. (2017). The world factbook: Field listing median age. Recuperado de https://www.cia.gov/library/ publications/the-world-factbook/fields/2177.html.

Child with a disability, 34 C.F.R. $§ 300.8$ (2018). Recuperado de https://www.ecfr.gov/cgi-bin/text-idx?SID=d9a1cf6b47e184115e9f0f12b6b673a4\&mc=true\&node=se34.2.300_18\&rgn=div8.

Constitución Española, art. 27 (2018). Recuperado de http://www.boe.es/legislacion/codigos/codigo.php?id=151_Constitucion_Espanola\&modo=1.

Determination of eligibility, 34 C.F.R. $§ 300.306$ (2018). Recuperado de https://www.ecfr.gov/cgi-bin/text-idx?SID=d9a1cf6b47e184115e9f0f12b6b673a4\&mc=true\&node=se34.2.300_1306\&rgn=div8.

Development, review, and revision of IEP, 34 C.F.R. § 300.324 (2018). Recuperado de https://www.ecfr.gov/cgi-bin/text-idx?-

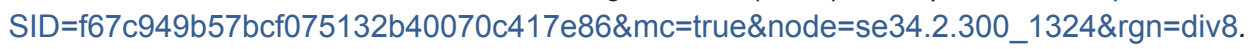


Discrimination prohibited, 34 C.F.R. $\S 100.3$ (2018). Recuperado de https://www.ecfr.gov/cgi-bin/text-idx?SID=c6ca34a92671c2ab55ab5c5925c58f1f\&mc=true\&node=se34.1.100_13\&rgn=div8.

Discrimination prohibited, 34 C.F.R. $§ 104.4$ (2018). Recuperado de https://www.ecfr.gov/cgi-bin/text-idx?SID=c6ca34a92671c2ab55ab5c5925c58f1f\&mc=true\&node=se34.1.104_14\&rgn=div8.

Elementary and Secondary Education Act of 1965 [as amended by the Every Student Succeeds Act of 2015], 20 U.S.C. § 6301 (2015). Recuperado de http://uscode.house.gov/view.xhtml?path=/prelim@title20/chapter70\&edition=prelim.

European Agency for Special Needs and Inclusive Education. (2017a). European Agency Statistics on Inclusive Education: 2014 Dataset Cross-Country Report. (J. Ramberg, A. Lénárt, and A. Watkins, eds.). Odense, Denmark. Recuperado de https://www.european-agency.org/resources/publications/european-agency-statistics-inclusive-education-2014-dataset-cross-country.

European Agency for Special Needs and Inclusive Education. (2017b). Who we are, What we focus on. Recuperado de https://www.european-agency.org/sites/default/files/about-us/AgencyFlyer2017-EN_A4_electronic.pdf.

European Agency for Special Needs and Inclusive Education. (n.d.). Spain - Overview. Recuperado de https://www. european-agency.org/country-information/spain/national-overview/complete-national-overview.

European Parliament. (2017). Inclusive education for learners with disabilities. Recuperado de http://www.europarl. europa.eu/RegData/etudes/STUD/2017/596807/IPOL_STU(2017)596807_EN.pdf.

Eurydice. (2015). Educational support and guidance. Recuperado de https://webgate.ec.europa.eu/fpfis/mwikis/eurydice/ index.php/Spain:Educational_Support_and_Guidance.

Eurydice. (2018). Spain: Funding in Education. Recuperado de https://eacea.ec.europa.eu/national-policies/eurydice/content/funding-education-79_en.

Evaluations and reevaluations, 34 C.F.R. $§ \S 300.301$ - 300.304 (2018). Recuperado de https://www.ecfr.gov/cgi-bin/text-

idx?SID=b6928d0cc3a88736e2dcbd2ff68ebde4\&mc=true\&node=sg34.2.300_1300.sg21\&rgn=div7.

Free Appropriate Public Education, 34 C.F.R. $\$ 300.101$ (2018). Recuperado de https://www.ecfr.gov/cgi-bin/text-idx?SID=5565b91e1ca135f456955cd8d50d3154\&mc=true\&node=se34.2.300_1101\&rgn=div8.

Grants to states, 34 C.F.R. $\S 300.700$ (2018). Recuperado de https://www.ecfr.gov/cgi-bin/text-idx?SID=5565b91e1ca$135 \mathrm{f} 456955 \mathrm{~cd} 8 \mathrm{~d} 50 \mathrm{~d} 3154 \& \mathrm{mc}=$ true $\&$ node=sp34.2.300.g\&rgn=div6.

Individualized Education Programs, 34 C.F.R. $\S \S 300.320$ - 300.323 (2018). Recuperado de https://www.ecfr.gov/cgi-bin/ text-idx?SID=f67c949b57bcf075132b40070c417e86\&mc=true\&node=sg34.2.300_1311.sg23\&rgn=div7.

Individualized Family Service Plan, 20 U.S.C. § 1436 (2004). Recuperado de http://uscode.house.gov/view.xhtml?path=/ prelim@title20/chapter33\&edition=prelim.

Individuals with Disabilities Act, 20 U.S.C. § 1400 (2004). Recuperado de http://uscode.house.gov/view.xhtml?path=/ prelim@title20/chapter33\&edition=prelim.

Membership, 34 C.F.R. $§ 300.168$ (2018). Recuperado de https://www.ecfr.gov/cgi-bin/retrieveECFR?gp=\&SID=ea682aa509df6044edc4527efb415b07\&mc=true\&n=pt34.2.300\&r=PART\&ty=HTML\#se34.2.300_1168.

Ministerio de Educación (2011). Plan de inclusión del alumnado con necesidades educativas especiales. Recuperado de www.mihijodown.com/adjuntos/.../29_1_plande.pdf.

Ministerio de Educación, Cultura y Deporte. (2013a). LOMCE paso a paso: Atención a la diversidad en la educación primaria. Recuperado de https://www.mecd.gob.es/dam/jcr:99b22cf2-ef7f-40dd-bfcd-9520beead9e6/lomced-pasoapaso-primaria-diversidad-v4.pdf.

Ministerio de Educación, Cultura y Deporte. (2013b). LOMCE paso a paso: Atención a la diversidad en la educación secundaria obligatoria y bachillerato. Recuperado de https://www.mecd.gob.es/dam/jcr:09a49003-ec3d-4093af30-d715ea35c9fb/lomced-pasoapaso-secundariaybac-diversidad-v51.pdf.

Ministerio de Educación, Cultura y Deporte. (2016). Education facts and figures: 2016/2017 School year. Recuperado de https://www.mecd.gob.es/servicios-al-ciudadano-mecd/dms/mecd/servicios-al-ciudadano-mecd/estadisticas/educacion/indicadores-publicaciones-sintesis/datos-cifras/Datosycifras1617ing.pdf.

Ministerio de Sanidad, Servicios Sociales e Igualdad. (2013). Plan nacional de acción para la inclusión social del Reino de España. Recuperado de http://www.eapn.es/publicaciones/173/plan-nacional-de-accion-para-la-inclusion-social-2013-2016.

National Center for Education Statistics. (2017). Children and youth with disabilities. Recuperado de https://nces.ed.gov/ programs/coe/indicator_cgg.asp.

National Center for Education Statistics. (2016). Digest of education statistics. Recuperado de https://nces.ed.gov/programs/ digest/d16/tables/dt16_204.60.asp.

Natural environments, 34 C.F.R. $§ 303.26$ (2018). Recuperado de https://www.ecfr.gov/cgi-bin/retrieveECFR?gp=\&SI$\mathrm{D}=902 \mathrm{c} 1492027 \mathrm{c} 47469323 \mathrm{df5} 1 \mathrm{fb} 596216 \& \mathrm{mc}=\operatorname{true} \& n=p t 34.2 .303 \& \mathrm{r}=$ PART\&ty=HTML\#se34.2.303_126. 
Organización de la Naciones Unidas para la Educación, la Ciencia, y la Cultura. (1994). Declaración de Salamanca. Recuperado de http://www.unesco.org/education/pdf/SALAMA_S.PDF.

Organization for Economic Co-operation and Development. (2003). Chapter 1 - Diversity, inclusion and equity: Insights from special needs provision. Education policy analysis (pp. 9-37). Recuperado de https://read.oecd-ilibrary.org/ education/education-policy-analysis-2003_epa-2003-en\#page4.

Organization for Economic Co-operation and Development. (2018a). Education spending [indicator]. doi: 10.1787/ca274bac-en (Accessed on 19 January 2018).

Organization for Economic Co-operation and Development. (2018b). Public spending on education [indicator]. doi: 10.1787/ f99b45d0-en (Accessed on 19 January 2018).

Parent and family engagement, 20 U.S.C. $§ 6318$ (2015). Recuperado de http://uscode.house.gov/view.xhtml?path=/ prelim@title20/chapter70\&edition=prelim.

Parent participation, 34 C.F.R. $§ 300.322$ (2018). Recuperado de https://www.ecfr.gov/cgi-bin/text-idx?SID=ea682aa509df6044edc4527efb415b07\&mc=true\&node=se34.2.300_1322\&rgn=div8.

Personnel qualifications, 34 C.F.R. $\S 300.156$ (2018). Recuperado de https://www.ecfr.gov/cgi-bin/retrieveECFR?gp=\&SI$\mathrm{D}=$ ea682aa509df6044edc4527efb415b07\&mc=true\&n=pt34.2.300\&r=PART\&ty=HTML\#se34.2.300_1156.

Real Patronato sobre Discapacidad. (2000). La realidad actual de la Atención Temprana en España. Recuperado de http:// sid.usal.es/idocs/F8/FDO26068/Atencion\%20Temprana.pdf.

Related services, 34 C.F.R. $§ 300.34$ (2018). Recuperado de https://www.ecfr.gov/cgi-bin/text-idx?SID=f67c949b57bcf075132b40070c417e86\&mc=true\&node=se34.2.300_134\&rgn=div8.

Transition Services, 20 U.S.C. § 1401 (2004). Recuperado de http://uscode.house.gov/view.xhtml?path=/prelim@title20/ chapter33\&edition=prelim.

TASH.(s.f.).Frequentlyaskedquestionsaboutinc/usiveeducation.Recuperado de https://tash.org/wp-content/uploads/2015/02/ TASH_Frequently-Asked-Questions-About-Inclusive-Education-4.pdf.

United Nations. (2006). United Nations convention on the rights of persons with disabilities. Recuperado de http://www. un.org/disabilities/documents/convention/convention_accessible_pdf.pdf.

United Nations Educational, Scientific and Cultural Organization. (1994). Declaración de Salamanca y marco de acción para las necesidades educativas especiales. Recuperado de http://www.unesco.org/education/pdf/SALAMA_S.PDF.

United Nations Educational, Scientific and Cultural Organization. (2009). Policy guidelines on inclusion in education. Recuperado de http://unesdoc.unesco.org/images/0017/001778/177849e.pdf.

United Nations Educational, Scientific and Cultural Organization. (2015). Education 2030: Incheon declaration and framework for action for the implementation of Sustainable Development Goal 4. Recuperado de http://uis.unesco.org/sites/default/files/documents/education-2030-incheon-framework-for-action-implementation-of-sdg4-2016-en_2.pdf.

United States Department of Education Office of Special Education and Rehabilitative Services. (2008).

Education and inclusion in the United States: A brief overview. Recuperado de http://www.ibe.unesco.org/National_Reports/ ICE_2008/usa_NR08.pdf.

United States Department of Education. (2017a). Budget fact sheet. Recuperado de https://ed.gov/about/overview/budget/ budget17/budget-factsheet.pdf.

United States Department of Education. (2017b). Special education fiscal year 2017 budget request. Recuperado de https:// www2.ed.gov/about/overview/budget/budget17/justifications/h-specialed.pdf.

United States Department of Heath and Human Services and United States Department of Education. (2015). Policy statement on inclusion of children with disabilities in early childhood programs. Recuperado de https://www2.ed.gov/ policy/speced/guid/earlylearning/joint-statement-full-text.pdf.

Universidad Internacional de Valencia. (2014). Trabajando la educación inclusive: La inclusión de los niños con necesidades educativas especiales. Recuperado de https://viu.hosting-mautic.com/guia-trabajando-la-educacion-inclusiva.

Use of funds, 20 U.S.C. $§ 1454$ (2004). Recuperado de http://uscode.house.gov/view.xhtml?path=/prelim@title20/chapter33\&edition=prelim.

Valle, J.M. (2012). La política supranacional : Un nuevo campo de conocimiento para abordar las políticas educativas en un mundo globalizado. Revista Española de Educación Comparada, 20, 109-144. https://doi.org/10.5944/reec.20.2012.7595.

What are the factors for determining equitable participation of children and teachers in private schools, 34 C.F.R. § 299.7 (2018). Recuperado de https://www.ecfr.gov/cgi-bin/text-idx?SID=5565b91e1ca135f456955cd8d50d3154\&mc=true\&node=se34.1.299_17\&rgn=div8.

WorldBankGroup.(2017).Expenditureoneducationas\%oftotalgovernmentexpenditure[Graph].

UNESCO Institute for Statistics. Recuperado de https://data.worldbank.org/indicator/SE.XPD.TOTL.GB.ZS?end=2014\&Iocations=MX-ES-US\&start=2010\&view=chart. 
Figura 1. Cuadro Comparativo de Educación Inclusiva: Categorías, Parámetros e Indicadores Elaboración personal a partir de las fuentes citadas en Anexo 3.

Figura 2. Cuadro Comparativo de Educación Inclusiva: Resultados por país: España y EEUU Elaboración personal a partir de la información citada en capítulo 2.

\section{Anexo 2. Abreviaturas}

BOE - Agencia Estatal Boletín Oficial del Estado

CCAA - Comunidades Autónomas

CE - Constitución Española

CFR -Code of Federal Regulations

CIA - Central Intelligence Agency

DOE - Department of Education

EASNIE - European Agency for Special Needs and Inclusive Education EEUU - Estados Unidos

ESSA - Every Student Succeeds Act

IDEA - Individuals with Disabilities Education Act

IEP - Individualized Education Program

INEE - Instituto Nacional de la Evaluación de la Educación

LOE - Ley Orgánica de la Educación

LOMCE - Ley Orgánica de la Mejora de la Calidad de la Educación

MECD - Ministerio de Educación, Cultura, y Deporte

NCES - National Center for Education Statistics

NEE - Necesidades Educativas Especiales

OCDE-Organización para la Cooperación y el Desarrollo Económicos

OECD - Organization of Economic Cooperation and Development

ONU - Organización de NacionesUnidas

OSERS - Office of Special Education and Rehabilitative Services

PES - Política Educativa Supranacional

RD - Real Decreto

SLD - Specific Learning Disabilities

TDAH-trastorno por déficit de atención e hiperactividad

UE - Unión Europea

UN - United Nations

UNESCO - United Nations Educational, Scientific, and Cultural Organization

USC - United States Code 
Anexo 3. Fuentes de los indicadores desde PE

\begin{tabular}{|c|c|}
\hline Indicadores & Fuentes \\
\hline Políticas de financiación & $\begin{array}{l}\text { UNESCO (1994) p.19; } \\
\text { UNESCO (2015) p.32, } 67\end{array}$ \\
\hline Recursos adecuados y equilibrados en escuelas públicas como privadas & UNESCO (1994) p.13 \\
\hline Acceso gratis & $\begin{array}{l}\text { UN (2006) p.14; } \\
\text { UNESCO (2015) p.7 }\end{array}$ \\
\hline Regulaciones que garantizar equidad y prevenir discriminación y desigualdad & $\begin{array}{l}\text { UN (2006) p.5-6, 13-14; } \\
\text { UNESCO (1994) p.11; } \\
\text { UNESCO (2015) p.45 }\end{array}$ \\
\hline Participación del individuo y familias en toma de decisiones, recursos y apoyo & $\begin{array}{c}\text { OECD (2003) p. } 27 ; \\
\text { UN (2006) p.2; } \\
\text { UNESCO (1994) p.37-38; } \\
\text { UNESCO (2009) p. } 18 ; \\
\text { UNESCO (2015) p.37 }\end{array}$ \\
\hline $\begin{array}{c}\text { Coordinación comunitaria y integración social a través de las esferas de salud, laboral, } \\
\text { programas de rehabilitación e infraestructura integrada }\end{array}$ & $\begin{array}{c}\text { OECD (2003) p.27; } \\
\text { UN (2006) p.12, 16; } \\
\text { UNESCO (1994) p.19, 38-39; } \\
\text { UNESCO (2009) p.14, 18; } \\
\text { UNESCO (2015) p.48 }\end{array}$ \\
\hline Asignación de recursos humanos, institucionales, logísticos, materiales, financieros, etc. & $\begin{array}{l}\text { UN (2006) p.5, 20; } \\
\text { UNESCO (1994) p.41-42; } \\
\text { UNESCO (2009) p.24; } \\
\text { UNESCO (2015) p.10, } 39\end{array}$ \\
\hline Políticas / leyes de inclusión educativa & $\begin{array}{l}\text { OECD (2003); UN (2006); } \\
\text { UNESCO (1994); } \\
\text { UNESCO (2009); } \\
\text { UNESCO (2015) }\end{array}$ \\
\hline $\begin{array}{l}\text { Políticas que respondan a la diversidad y las necesidades como discapacidad, } \\
\text { diversidad lingüística, personas indígenas, minorías, y los pobres. }\end{array}$ & $\begin{array}{c}\text { UN (2006) p.2, 20; } \\
\text { UNESCO (1994) p.6; } \\
\text { UNESCO (2009) p.8; } \\
\text { UNESCO (2015) p.25, } 30\end{array}$ \\
\hline Formación inicial en universidad & $\begin{array}{c}\text { OECD (2003) p.25; } \\
\text { UNESCO (1994) p.29; } \\
\text { UNESCO (2009) p.17, 20-21 }\end{array}$ \\
\hline
\end{tabular}




\begin{tabular}{|c|c|}
\hline Formación colaborativo en el servicio & $\begin{array}{c}\text { OECD (2003) p.25; } \\
\text { UN (2006) p.5, 15, 16; } \\
\text { UNESCO (1994) p.13, 28; } \\
\text { UNESCO (2009) p.17, 20-21; } \\
\text { UNESCO (2015) p.30, } 32\end{array}$ \\
\hline Requisitos para el proceso de referencia & UNESCO (1994) p.22 \\
\hline Requisitos para el proceso de evaluación & $\begin{array}{l}\text { UN (2006) p.16; } \\
\text { UNESCO (1994) p.22 }\end{array}$ \\
\hline Definición de discapacidad o necesidades educativas especiales & $\begin{array}{l}\text { EASNIE (2017a) p.9; } \\
\text { UN (2006) p.1; } \\
\text { UNESCO (1994) p.6; } \\
\text { UNESCO (2009) p.23 }\end{array}$ \\
\hline Criterios de elegibilidad para determinar si una necesidad especial está presente & $\begin{array}{l}\text { European Parliament } \\
\text { (2017) p.37 }\end{array}$ \\
\hline Participación requerida en escuelas ordinarias & $\begin{array}{c}\text { OECD (2003) p.11; } \\
\text { UN (2006) p.15; } \\
\text { UNESCO (1994) p.12, } 17-18\end{array}$ \\
\hline Niveles de inclusión relacionadas con la gravedad; individualizada & $\begin{array}{l}\text { UN (2006) p.15; } \\
\text { UNESCO (1994) p.7, 13; } \\
\text { UNESCO (2009) p.16 }\end{array}$ \\
\hline $\begin{array}{c}\text { Incluye servicios de desarrollo, psicológicos, físicos, ocupacionales, de } \\
\text { comunicación y auditivos / visuales en la escuela }\end{array}$ & $\begin{array}{c}\text { OECD (2003) p.26; } \\
\text { UN (2006) p.16; } \\
\text { UNESCO (1994) p.31-32; } \\
\text { UNESCO (2009) p.20 }\end{array}$ \\
\hline $\begin{array}{l}\text { Pautas de documentación formal y concisa para la implementación de } \\
\text { servicios individualizada }\end{array}$ & $\begin{array}{c}\text { OECD (2003) p.25; } \\
\text { UN (2006) p.15, 16; } \\
\text { UNESCO (2009) p.19, } 26\end{array}$ \\
\hline $\begin{array}{l}\text { Ayudas técnicas que facilitan la comunicación, la movilidad, y el aprendizaje } \\
\text { (apoyo que requieran) }\end{array}$ & $\begin{array}{c}\text { OECD (2003) p.28; } \\
\text { UN (2006) p.3, 4; } \\
\text { UNESCO (1994) p.22-23 }\end{array}$ \\
\hline Pronto identificación de edades 0-6 años & $\begin{array}{l}\text { UNESCO (1994) p.33; } \\
\text { UNESCO (2009) p.15; } \\
\text { UNESCO (2015) p.38 }\end{array}$ \\
\hline
\end{tabular}




\begin{tabular}{|c|c|}
\hline Accesibilidad a servicios regulada por ley & $\begin{array}{l}\text { UNESCO (2009) p.17; } \\
\text { UNESCO (2015) p.40 }\end{array}$ \\
\hline Servicios provistos en entorno natural con colaboración familiar y comunitaria & $\begin{array}{c}\text { UNESCO (1994) p.33-34; } \\
\text { UNESCO (2009) p.12; } \\
\text { UNESCO (2015) p.39 }\end{array}$ \\
\hline Regulación de programas de transición, formación profesional, e inserción laboral & $\begin{array}{c}\text { UNESCO (1994) p.33, 35; } \\
\text { UNESCO (2015) p.52 }\end{array}$ \\
\hline
\end{tabular}

